\title{
Galaxy properties in clusters
}

\section{Backsplash galaxies}

\author{
H. Muriel ${ }^{1,2}$ and V. Coenda ${ }^{1,2}$ \\ ${ }^{1}$ Instituto de Astronomía Teórica y Experimental IATE, CONICET, Laprida 922, X5000BGR Córdoba, Argentina \\ 2 Observatorio Astronómico, Universidad Nacional de Córdoba, Laprida 854, X5000BGR Córdoba, Argentina \\ e-mail: [hernan;vcoenda]@mail.oac.uncor.edu
}

Received 6 June 2013 / Accepted 14 February 2014

\begin{abstract}
Aims. We explore the properties of galaxies on the outskirts of clusters and their dependence on recent dynamical history in order to understand the real impact that the cluster core has on the evolution of galaxies.

Methods. We analyse the properties of more than 1000 galaxies brighter than $M_{0.1 r}=-19.6$ on the outskirts of 90 clusters $(1<$ $r / r_{\text {vir }}<2$ ) in the redshift range $0.05<z<0.10$. Using the line of sight velocity of galaxies relative to the cluster's mean, we selected low and high velocity subsamples. Theoretical predictions indicate that a significant fraction of the first subsample should be backsplash galaxies, that is, objects that have already orbited near the cluster centre. A significant proportion of the sample of high relative velocity (HV) galaxies seems to be composed of infalling objects.

Results. Our results suggest that, at fixed stellar mass, late-type galaxies in the low-velocity (LV) sample are systematically older, redder, and have formed fewer stars during the last 3 Gyrs than galaxies in the HV sample. This result is consistent with models that assume that the central regions of clusters are effective in quenching the star formation by means of processes such as ram pressure stripping or strangulation. At fixed stellar mass, LV galaxies show some evidence of having higher surface brightness and smaller size than HV galaxies. These results are consistent with the scenario where galaxies that have orbited the central regions of clusters are more likely to suffer tidal effects, producing loss of mass as well as a re-distribution of matter towards more compact configurations. Finally, we found a higher fraction of ET galaxies in the LV sample, supporting the idea that the central region of clusters of galaxies may contribute to the transformation of morphological types towards earlier types.
\end{abstract}

Key words. galaxies: clusters: general - galaxies: evolution

\section{Introduction}

The relative importance of nature vs. nurture (heredity vs. environment) in the formation and evolution of galaxies has been increasingly discussed in recent years. There is consensus that both are important and De Lucia et al. (2012) have shown that these two elements of galaxy evolution are inevitably intertwined. In the downsizing scenario, stars in more massive galaxies tend to have formed earlier and over a short period of time (Neistein et al. 2006). This effect in the early stages of galaxy formation suggests that the initial conditions (nature) are important.

The strong correlation between the present-day galaxy properties and the environment is well known (see for instance Blanton et al. 2005). In the hierarchical clustering scenario, the structures form from the bottom up, via the merging of small objects into successively more massive systems. In this scenario, the environment in which galaxies evolve can vary substantially with time. Extreme cases are clusters of galaxies, which favour a great number of physical processes that could modify the evolutionary history of galaxies. In this environment, gravitational phenomena such as the interactions and merger of galaxies are frequent and can produce morphological transformations (Moore et al. 1998). Moreover, the presence of the hot intracluster medium favours processes like ram pressure (Gunn \& Gott 1972; Abadi et al. 1999) or strangulation (Larson et al. 1980), which can affect both the gaseous content of galaxies and their star formation history (Fujita \& Nagashima 1999).
Galaxies can also suffer the stripping of gas, stars, and dark matter as a result of interaction with the cluster potential (Moore et al. 1999). These aspects make clusters of galaxies ideal objects for evaluating the processes involved in the evolution of galaxies. Recently it has been suggested that group environment also plays a key role in present-day galaxy properties (e.g. Wilman et al. 2009). Poggianti et al. (2009) analysed a combined sample of nearby and high redshift clusters of galaxies and found that the spiral and S0 fractions have evolved more strongly in less massive clusters, suggesting that morphological evolution since redshift 1 is more pronounced in low-mass clusters. Rasmussen et al. (2012) found that the fraction of star-forming group members is suppressed relative to the field, mirroring results for massive clusters (see also Mahajan 2013).

The well-known morphological segregation of galaxies into clusters (Dressler 1980) does not imply that the local density or distance to the cluster centre remains unchanged. Since the typical time-scale of galaxy orbits in a cluster is of the order of a few Gyrs (McCarthy et al. 2008), these galaxies must have passed close to the centre of the cluster since their infall. Moreover, it is to be expected that a fraction of the galaxies that have been close to the cluster centre would today be on the outskirts. Using high-resolution $N$-body simulations Gill et al. (2005) found that approximately half of the galaxies that at present have clustocentric distances between 1 and 2 virial radii were in the past close to the cluster centre. These systems are usually called backsplash galaxies. More recently, Mahajan et al. (2011) investigated how, 
at a given projected clustocentric radius, the stellar mass and star formation activity of a galaxy depend on its line-of-sight velocity relative to the cluster's mean velocity $\left(v_{\text {los }}\right)$. Based on $\mathrm{N}$-body simulations, they found that the fraction of backsplash objects depends on the velocity threshold: one-third at projected radii slightly greater than the virial radius and $\left|v_{\text {los }}\right|<\sigma$ (or one half when $\left|v_{\operatorname{los}}\right| \ll \sigma$ ), where $\sigma$ is the velocity dispersion of the cluster. If the backsplash and the infalling populations can be identified separately, the effects on the galaxy properties caused by the core of clusters can be distinguished from the preprocessing in groups. Mahajan et al. (2011) analysed a sample of clusters selected from a group catalogue and found that star formation in galaxies is almost completely quenched in a single passage through the cluster. The role of the backsplash scenario in the evolution of galaxies is also tested in Rines et al. (2005), Pimbblet et al. (2006), and Aguerri \& Sánchez-Janssen (2010).

Based on the backsplash scenario, in this paper we address the question of the influence of cluster environment in galaxy evolution. For this purpose, we use the sample of galaxies in clusters selected by Coenda \& Muriel (2009; hereafter Paper I) plus a new subsample of X-ray clusters obtained by crosscorrelating the Northern ROSAT All-Sky Galaxy Cluster Survey (NORAS; Bohringer et al. 2000, hereafter B00) with the Sloan Digital Sky Survey (SDSS; York et al. 2000). We have analysed a wide range of properties of high- and low-velocity subsamples of cluster members with clustocentric distances $1<r / r_{\text {vir }}<2$ in the redshift range $0.05<z<0.10$. The results are analysed in the framework of models of the formation and evolution of galaxies.

This paper is organised as follows: in Sect. 2 we describe the samples of clusters and galaxies. Galaxy properties and their dependence on environment are analysed in Sect. 3. In Sect. 4 we discuss the results and outline our conclusions.

\section{The sample}

\subsection{The cluster sample}

Clusters of galaxies used in this paper were selected from samples identified using different techniques. Two are based on the $\mathrm{X}$-ray emission produced by the hot gas in the intra-cluster medium, the ROSAT-SDSS Galaxy Cluster Survey of Popesso et al. (2004; hereafter P04) and NORAS of B00; the other one makes use of the well-known segregation of galaxies, according to which clusters are mostly populated by red early-type galaxies and the presence of a dominant galaxy in the cluster centre: the MaxBCG Catalogue of Koester et al. (2007b; hereafter K07).

The ROSAT-SDSS catalogue of P04 comprises 114 galaxy clusters detected in the ROSAT All Sky Survey (RASS) lying in the area surveyed by the SDSS in February 2003. This X-rayselected catalogue includes clusters with masses from $10^{12.5} \mathcal{M}_{\odot}$ to $10^{15} \mathcal{M}_{\odot}$ in the redshift range $0.002 \leq z \leq 0.45$ and X-ray luminosity $L_{\mathrm{x}}(0.1-2.4 \mathrm{keV}) \leq 30.14 \times 10^{44} \mathrm{erg} \mathrm{s}^{-1}$. On the other hand, NORAS covers the celestial region of declination $\delta \geq 0^{\circ}$ and galactic latitude $\left|b_{\mathrm{II}}\right| \geq 20^{\circ}$. The $\mathrm{B} 00$ sample comprises 378 $\mathrm{X}$-ray sources that show extended X-ray emission in the first processing of the ROSAT All-Sky Survey with redshift $z \leq 0.46$ and $\mathrm{X}$-ray luminosity $L_{x}(0.1-2.4 \mathrm{keV}) \leq 32.56 \times 10^{44} \mathrm{erg} \mathrm{s}^{-1}$.

In paper I, we selected an X-ray sample of 49 clusters of galaxies from $\mathrm{P} 04$ in the redshift range $0.05<z<0.14$, labelled as C-P04-I. Galaxies in these clusters were identified using the Main Galaxy Sample (MGS; Strauss et al. 2002) of the Fifth Data Release (DR5; Adelman-McCarthy et al. 2007) of SDSS, which includes spectroscopic redshifts down to a
Petrosian (1976) magnitude $r=17.77$. In this paper, we expand the X-ray cluster sample using the cross-correlation between NORAS and SDSS. We indentify a subsample from B00, which we labelled C-B00-I, using the MGS of the Seventh Data Release (DR7; Abazajian et al. 2009) of SDSS. This subsample comprises 55 galaxy clusters in the redshift range $0.05<z<$ 0.14 (the clusters are listed in Table 1).

The optical MaxBCG catalogue provides sky locations, photometric redshift estimates, and richness for 13823 clusters. Details of the selection algorithm and catalogue properties are published in Koester et al. (2007) and K07, respectively. The MaxBCG selection relies on the observation that the galaxy population of rich clusters is dominated by bright red galaxies tightly clustered in colour (the E/S0 ridgeline). Since these galaxies are old, passively evolving stellar populations, their $g-r$ colours closely reflect their redshifts.

Galaxies in optically-selected MaxBCG clusters belong to the C-K07-I sample of Paper I. It consists of selected clusters from the catalogue of K07, using the MGS of DR5. For the C-K07-I sample, we apply a restriction in the richness, selecting clusters with $N_{\text {gal }} \geq 20$ in order to have cluster masses comparable to those in the C-P04-I sample. The C-K07-I sample comprises 209 regular galaxy clusters in the redshift range $0.05<z<0.14$.

The selection of cluster members and the estimation of the physical properties of C-B00-I clusters are made using the same method that we applied to C-P04-I and C-K07-I in Paper I. To select cluster members, we use the friends-of-friends ( $f o f$ ) algorithm developed by Huchra \& Geller (1982) with percolation linking length values according to Díaz et al. (2005): density contrast of $\delta \rho / \rho=315$, line-of-sight linking length of $V_{0}=200 \mathrm{~km} \mathrm{~s}^{-1}$, and a projected separation $D_{0}=0.189 \mathrm{Mpc}$. As a result, we obtain a list of substructures for each field with at least ten members identified by fof. The second step consists of an eyeball examination of the structure detected by fof. From the redshift distribution of galaxies, we determine the line-ofsight extension of each cluster and therefore the cluster members. Through visual inspection, we exclude systems that have two or more close substructures of similar size in the plane of the sky and/or in the redshift distribution. Once the members of each cluster are selected, we compute physical properties of the clusters using methods described by Beers et al. (1990). These include the line-of-sight velocity dispersion $\sigma$, virial radius, and mass.

The final sample used in this paper is a composite of 27 C-P04-I, 32 C-K07-I, and 31 C-B00-I cluster of galaxies, that we labelled PKB, which comprises 90 clusters in the redshift range $0.05<z<0.10$. Figure 1 shows the distributions of physical properties of the clusters (black lines) of our final sample and their median values are shown in Table 2.

\subsection{The galaxy sample}

Properties of galaxies can be affected by the environment. Among the most commonly studied are morphology, colours, luminosities, and sizes. Throughout this work we use two sets of galaxy properties. The first set is based on the photometric properties of galaxies: the Petrosian $r$-band absolute magnitude $M_{0.1} r$, the radius that encloses $50 \%$ of the Petrosian flux $r_{50}$, the $r$-band surface brightness $\mu_{50}$ computed inside $r_{50}$, and the ${ }^{0.1}(g-r)$. Colours are computed using model magnitudes instead of Petrosian magnitudes. Model magnitudes are calculated using the best-fit parameters in the $r$-band, and the same model is applied to the other bands; the light is therefore measured 
H. Muriel and V. Coenda: Galaxy properties in clusters. II.

Table 1. The C-B00-I cluster sample.

\begin{tabular}{|c|c|c|c|c|c|c|}
\hline $\begin{array}{c}\alpha(\mathrm{J} 2000.0) \\
{[\mathrm{h} \mathrm{m} \mathrm{s}]}\end{array}$ & $\begin{array}{c}\delta(\mathrm{J} 2000.0) \\
{\left[{ }^{\circ},{ }^{\prime}{ }^{\prime}\right]}\end{array}$ & $z$ & $\begin{array}{l}\sigma \\
{\left[\mathrm{km} \mathrm{s}^{-1}\right]}\end{array}$ & $\begin{array}{l}\mathcal{M}_{\text {vir }} \\
{\left[h^{-1} \mathcal{M}_{\odot}\right]}\end{array}$ & $\begin{array}{l}R_{\mathrm{vir}} \\
{\left[h^{-1} \mathrm{Mpc}\right]}\end{array}$ & $\begin{array}{l}R_{200} \\
{\left[h^{-1} \mathrm{Mpc}\right]}\end{array}$ \\
\hline 072550.8 & +412252.7 & 0.110 & 430.52 & $0.231 \times 10^{15}$ & 1.783 & 1.065 \\
\hline 074637.3 & +310049.0 & 0.058 & 707.25 & $0.385 \times 10^{15}$ & 1.103 & 1.750 \\
\hline 075942.8 & +540005.8 & 0.102 & 472.30 & $0.224 \times 10^{15}$ & 1.437 & 1.169 \\
\hline 080543.3 & +454125.1 & 0.135 & 974.78 & $0.889 \times 10^{15}$ & 1.341 & 2.412 \\
\hline 081102.4 & +164405.6 & 0.092 & 734.88 & $0.480 \times 10^{15}$ & 1.275 & 1.818 \\
\hline 082101.8 & +075157.6 & 0.110 & 454.99 & $0.210 \times 10^{15}$ & 1.452 & 1.126 \\
\hline 082838.9 & +302540.8 & 0.050 & 827.01 & $0.740 \times 10^{15}$ & 1.552 & 2.046 \\
\hline 084437.5 & +425910.0 & 0.054 & 375.80 & $0.915 \times 10^{14}$ & 0.928 & 0.930 \\
\hline 092414.0 & +140952.9 & 0.140 & 531.81 & $0.357 \times 10^{15}$ & 1.811 & 1.316 \\
\hline 100634.2 & +255532.2 & 0.117 & 469.32 & $0.199 \times 10^{15}$ & 1.292 & 1.161 \\
\hline 101643.5 & +244607.7 & 0.173 & 1229.69 & $0.287 \times 10^{16}$ & 2.719 & 3.043 \\
\hline 101959.8 & +405914.3 & 0.092 & 901.98 & $0.726 \times 10^{15}$ & 1.280 & 2.232 \\
\hline 103135.0 & +350317.6 & 0.122 & 512.04 & $0.191 \times 10^{15}$ & 1.046 & 1.267 \\
\hline 103445.0 & $+3041 \quad 17.5$ & 0.137 & 744.20 & $0.641 \times 10^{15}$ & 1.659 & 1.841 \\
\hline 104043.9 & +395653.2 & 0.137 & 1291.03 & $0.218 \times 10^{16}$ & 1.877 & 3.194 \\
\hline 110918.8 & +413344.6 & 0.076 & 640.30 & $0.353 \times 10^{15}$ & 1.233 & 1.584 \\
\hline 111138.5 & +405032.6 & 0.075 & 742.21 & $0.594 \times 10^{15}$ & 1.545 & 1.836 \\
\hline 112214.5 & +671246.4 & 0.055 & 329.25 & $0.673 \times 10^{14}$ & 0.890 & 0.815 \\
\hline 112313.2 & +1935 58.6 & 0.104 & 784.16 & $0.810 \times 10^{15}$ & 1.888 & 1.940 \\
\hline 114345.5 & +462056.4 & 0.114 & 496.34 & $0.290 \times 10^{15}$ & 1.688 & 1.228 \\
\hline 115518.5 & +232427.0 & 0.143 & 1257.93 & $0.331 \times 10^{16}$ & 3.002 & 3.113 \\
\hline 115657.8 & +241529.2 & 0.139 & 899.86 & $0.118 \times 10^{16}$ & 2.096 & 2.227 \\
\hline 120147.6 & +580203.8 & 0.103 & 836.92 & $0.100 \times 10^{16}$ & 2.049 & 2.071 \\
\hline 120300.5 & +283450.9 & 0.133 & 739.39 & $0.312 \times 10^{15}$ & 0.818 & 1.830 \\
\hline 121030.6 & +052122.3 & 0.077 & 642.15 & $0.410 \times 10^{15}$ & 1.424 & 1.589 \\
\hline 122512.0 & +321344.8 & 0.061 & 501.81 & $0.240 \times 10^{15}$ & 1.365 & 1.242 \\
\hline 122728.0 & +084944.4 & 0.090 & 952.88 & $0.123 \times 10^{16}$ & 1.941 & 2.358 \\
\hline 122959.2 & +114720.8 & 0.085 & 878.40 & $0.125 \times 10^{16}$ & 2.331 & 2.173 \\
\hline 130345.6 & +191617.4 & 0.064 & 715.15 & $0.443 \times 10^{15}$ & 1.240 & 1.770 \\
\hline 133934.8 & +183043.9 & 0.114 & 957.25 & $0.130 \times 10^{16}$ & 2.028 & 2.369 \\
\hline 134148.8 & +262245.1 & 0.075 & 684.31 & $0.566 \times 10^{15}$ & 1.732 & 1.693 \\
\hline 134853.0 & +263544.2 & 0.063 & 860.93 & $0.105 \times 10^{16}$ & 2.038 & 2.130 \\
\hline 134921.6 & +280613.0 & 0.076 & 820.02 & $0.108 \times 10^{16}$ & 2.298 & 2.029 \\
\hline 135145.6 & +462200.5 & 0.062 & 571.32 & $0.345 \times 10^{15}$ & 1.518 & 1.414 \\
\hline 135408.6 & +145101.1 & 0.126 & 621.21 & $0.313 \times 10^{15}$ & 1.161 & 1.537 \\
\hline 141334.0 & +434024.2 & 0.089 & 612.68 & $0.310 \times 10^{15}$ & 1.184 & 1.516 \\
\hline 141551.6 & +001532.0 & 0.126 & 466.11 & $0.111 \times 10^{15}$ & 0.731 & 1.153 \\
\hline 142135.5 & +493306.8 & 0.072 & 639.39 & $0.374 \times 10^{15}$ & 1.311 & 1.582 \\
\hline 142352.4 & +401542.8 & 0.082 & 433.13 & $0.156 \times 10^{15}$ & 1.189 & 1.072 \\
\hline 142828.1 & +565258.4 & 0.107 & 791.82 & $0.934 \times 10^{15}$ & 2.135 & 1.959 \\
\hline 143107.1 & +253819.7 & 0.096 & 880.42 & $0.987 \times 10^{15}$ & 1.826 & 2.178 \\
\hline 144218.4 & +221817.3 & 0.096 & 568.21 & $0.402 \times 10^{15}$ & 1.783 & 1.406 \\
\hline 145307.2 & +215340.9 & 0.117 & 920.46 & $0.152 \times 10^{16}$ & 2.571 & 2.278 \\
\hline 145431.4 & +183831.2 & 0.059 & 645.70 & $0.501 \times 10^{15}$ & 1.724 & 1.598 \\
\hline 151011.7 & +333052.9 & 0.113 & 1214.50 & $0.249 \times 10^{16}$ & 2.417 & 3.005 \\
\hline 151106.3 & +180237.3 & 0.115 & 626.88 & $0.296 \times 10^{15}$ & 1.081 & 1.551 \\
\hline 151845.6 & +61352.3 & 0.102 & 799.14 & $0.925 \times 10^{15}$ & 2.076 & 1.977 \\
\hline 152040.3 & +483937.4 & 0.073 & 743.10 & $0.543 \times 10^{15}$ & 1.410 & 1.839 \\
\hline 153948.7 & +304302.3 & 0.097 & 778.23 & $0.668 \times 10^{15}$ & 1.582 & 1.926 \\
\hline 155820.6 & +271336.8 & 0.089 & 1118.92 & $0.248 \times 10^{16}$ & 2.846 & 2.769 \\
\hline 162031.7 & +295343.1 & 0.097 & 866.98 & $0.108 \times 10^{16}$ & 2.058 & 2.145 \\
\hline 165423.3 & +233411.6 & 0.058 & 519.73 & $0.289 \times 10^{15}$ & 1.533 & 1.286 \\
\hline 170948.8 & +342626.2 & 0.086 & 1094.27 & $0.181 \times 10^{16}$ & 2.168 & 2.708 \\
\hline 171757.4 & +323233.4 & 0.107 & 959.81 & $0.111 \times 10^{16}$ & 1.729 & 2.375 \\
\hline 172218.7 & +304408.2 & 0.047 & 576.22 & $0.271 \times 10^{15}$ & 1.172 & 1.426 \\
\hline
\end{tabular}

consistently through the same aperture in all bands. We exclude galaxies with $r_{50}<2^{\prime \prime}$ in order to reduce the effect of the PSF on galaxy sizes. Moreover, Petrosian quantities are not affected by seeing effects in our redshift range (Hyde \& Bernardi 2009). In addition, we correct the magnitudes and sizes by sky level using SDSS simulated galaxies ${ }^{1}$. Finally, we estimate the restframe radius $r_{50}$ in $r$-band as in Hyde \& Bernardi (2009). The maximum correction in magnitude and size are 0.03 and 0.1 ,

1 http://www.sdss.org/dr6/products/catalogs/index.html 
Table 2. Sample name (Col. 1), physical properties (Cols. 2-5), redshift ranges (Col. 6), and total number of clusters (Col. 7).

\begin{tabular}{|c|c|c|c|c|c|c|}
\hline Sample & $\begin{array}{c}\sigma \\
{\left[\mathrm{km} \mathrm{s}^{-1}\right]}\end{array}$ & $\begin{array}{c}R_{200} \\
{\left[h^{-1} \mathrm{Mpc}\right]}\end{array}$ & $\begin{array}{c}\mathcal{M}_{\text {vir }} \\
{\left[h^{-1} \mathcal{M}_{\odot}\right]}\end{array}$ & $\begin{array}{c}R_{\mathrm{vir}} \\
{\left[h^{-1} \mathrm{Mpc}\right]}\end{array}$ & Redshift & Cluster No. \\
\hline $\begin{array}{l}\text { C-B00-I } \\
\text { Median } \\
\text { Range }\end{array}$ & $\begin{array}{c}820 \\
329-1291\end{array}$ & $\begin{array}{c}2.03 \\
0.81-3.19\end{array}$ & $\begin{array}{c}9 \times 10^{14} \\
7 \times 10^{13}-3 \times 10^{15}\end{array}$ & $\begin{array}{c}1.83 \\
0.73-3.00\end{array}$ & $0.05 \leq z \leq 0.14$ & 55 \\
\hline $\begin{array}{l}\text { C-P04-I } \\
\text { Median } \\
\text { Range }\end{array}$ & $\begin{array}{c}715 \\
332-1060\end{array}$ & $\begin{array}{c}1.77 \\
0.82-2.62\end{array}$ & $\begin{array}{c}7 \times 10^{14} \\
5 \times 10^{13}-2 \times 10^{15}\end{array}$ & $\begin{array}{c}1.75 \\
0.71-2.56\end{array}$ & $0.05 \leq z \leq 0.14$ & 49 \\
\hline $\begin{array}{l}\text { C-K07-I } \\
\text { Median } \\
\text { Range }\end{array}$ & $\begin{array}{c}675 \\
212-1272 \\
\end{array}$ & $\begin{array}{c}1.67 \\
0.53-3.15\end{array}$ & $\begin{array}{c}6 \times 10^{14} \\
3 \times 10^{13}-2 \times 10^{15}\end{array}$ & $\begin{array}{c}1.59 \\
0.76-2.88\end{array}$ & $0.05 \leq z \leq 0.14$ & 207 \\
\hline $\begin{array}{l}\text { PKB } \\
\text { Median } \\
\text { Range }\end{array}$ & $\begin{array}{c}707 \\
231-1119\end{array}$ & $\begin{array}{c}1.75 \\
0.53-2.71\end{array}$ & $\begin{array}{c}6 \times 10^{14} \\
3 \times 10^{13}-3 \times 10^{15}\end{array}$ & $\begin{array}{c}1.64 \\
0.71-2.89\end{array}$ & $0.05 \leq z \leq 0.10$ & 90 \\
\hline
\end{tabular}
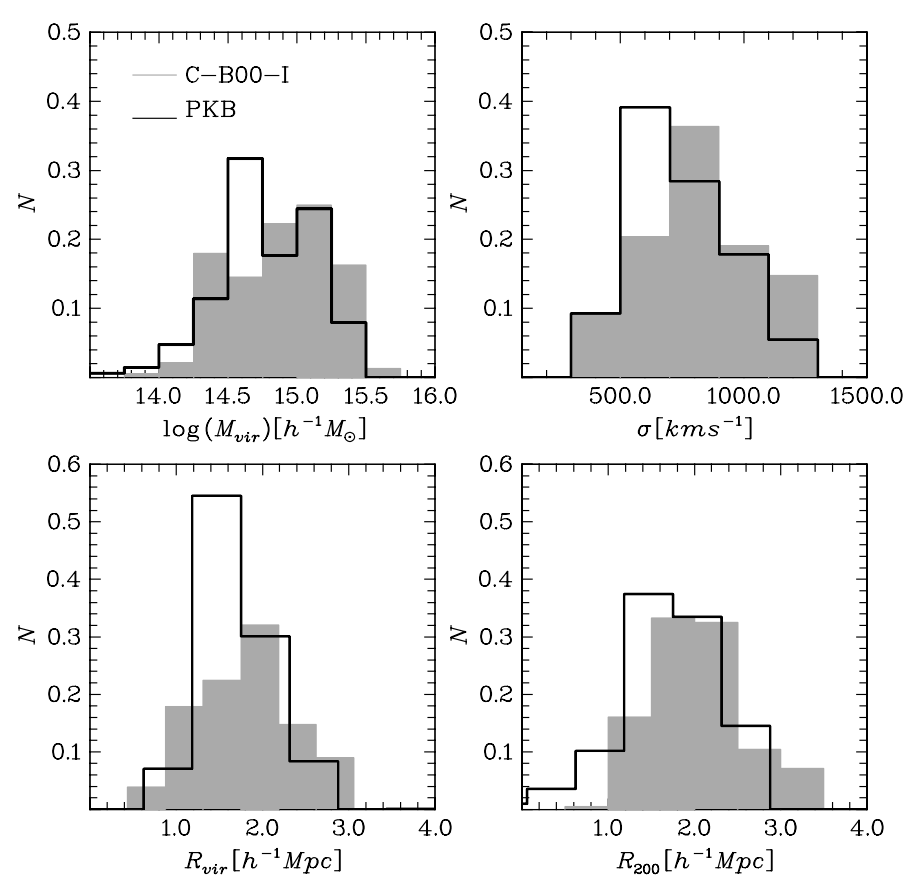

Fig. 1. Distributions of physical properties of the clusters for the PKB sample (black lines) in the redshift range $0.05<z<0.10$ and the C-B00-I sample (grey lines) in the redshift range $0.05<z<0.14$.

respectively. For more details see Paper I. It should be noted that we are using galaxies from different SDSS data releases. Based on the information in the SDSS documentation pages and a test with a random sample of galaxies we found that the impact of this inhomogeneity in the results of our work are minimal.

As in Paper I, in the analyses below we classify galaxies into early and late types according to their $r$-band concentration parameter defined as the ratio between the radii that encloses $90 \%$ and $50 \%$ of the Petrosian flux, $C=r_{90} / r_{50}$. The concentration index is known to correlate with the morphological type and can be used to separate early-type (E, S0, and Sa) from late-type (Sb, Sc, and Irr) galaxies (Shimasaku et al. 2001; Strateva et al. 2001; Park \& Choi 2005; Deng 2013). Typically, early-type galaxies have $C>2.5$, while for late-types $C<2.5$.

To perform a fair comparison, we weight each galaxy in our computations by $1 / V \max$ (Schmidt 1968) in order to compensate for the limitations of our flux-limited sample. Galaxy magnitudes used throughout this paper have been corrected for galactic extinction following Schlegel et al. (1998), and absolute magnitudes have been computed assuming $\Omega_{0}=0.3, \Omega_{\Lambda}=0.7$, and $H_{0}=70 h \mathrm{~km} \mathrm{~s}^{-1} \mathrm{Mpc}^{-1}$, and $K$-corrected using the method of Blanton et al. (2003, KCORRECT version 4.1). All magnitudes are in the $\mathrm{AB}$ system.

\subsection{Ages, metallicities, and stellar masses}

Spectral synthesis codes compare galaxy data with models by combining libraries of stellar spectra and prescriptions for the star formation and chemical histories. The implementation of these codes allows the estimation of the stellar masses, the mean ages $(\tau)$, and the metallicities $(Z)$ of the stellar population of galaxies. Rakos et al. (2007) analysed galaxies in Coma and Abell 1185, finding a clear correlation between $\tau$ and $Z$ with both the stellar mass of galaxies and the environment. They found a significant correlation between galaxy mean age and clustocentric distance, such that older galaxies inhabit the core. Ellison et al. (2009) analysed a large sample of cluster galaxies with nebular metallicities and found that galaxies in clusters have higher values of $Z$ relative to similar galaxies in low-density environments. They also found that galaxies in locally rich environments have higher median metallicities than those in locally poor environments. Although they do not find any correlation between $Z$ and the cluster properties, their results suggest that galaxies have higher metallicities in the core of clusters than on the outskirts.

Tojeiro et al. (2009) applied a stellar population model to the SDSS final data release using the VESPA code (Tojeiro et al. 2007). This code is based on the construction of galaxy histories from synthetic models. For the modelling of the single stellar population (SSP), the authors use the models of Bruzual \& Charlot (2003) and Maraston (2005). For the dust modelling, the code assumes that stars of all ages are affected in the same way and two different dust extinction models are considered. For the whole sample of cluster members, we use the VESPA code to compute the stellar mass, the mass-weighted age and the massweighted metallicity. The last two quantities are computed using two equations from Tojeiro \& Percival (2010),

$$
\begin{aligned}
& \left\langle t_{\text {age }}\right\rangle=\frac{\sum_{i} t_{i} x_{i}}{\sum_{i} x_{i}} \\
& \langle Z\rangle=\frac{\sum_{i} Z_{i} x_{i}}{\sum_{i} x_{i}},
\end{aligned}
$$

where $t_{i}\left(Z_{i}\right)$ is the mean age (metallicity) and $x_{i}$ is the star formation fraction in the age bin $i$. To test if star formation is 
H. Muriel and V. Coenda: Galaxy properties in clusters. II.

Table 3. Number of galaxies in each of the subsamples analysed and the total number of galaxies.

\begin{tabular}{ccccc}
\hline \hline ET-LV & LT-LV & ET-HV & LT-HV & Total \\
\hline 454 & 187 & 275 & 144 & 1060 \\
\hline
\end{tabular}

induced during the infall of galaxies into the clusters, we also compute the star formation fraction (SFF) during the last $3 \mathrm{Gyr}$. For our analysis, we adopt the RunID 2 of Tojeiro et al. (2009), which considers the SSP of Bruzual \& Charlot (2003) and a twoparameter model for the dust.

\subsection{The selection of backsplash galaxies}

We have stacked all the cluster members in the plane $r / r_{\text {vir }}$ $\left|v_{\text {los }}\right| / \sigma$ where the clustocentric distance $r$ is normalised to $r_{\text {vir }}$ and the absolute line of sight velocity (relative to the cluster) $\left|v_{\text {los }}\right|$ is normalised to the cluster velocity dispersion $\sigma$. Gill et al. (2005) suggested that $50 \%$ of the galaxies in the interval 1-2 virial radii of clusters are backsplash systems typically have $v_{\text {los }}<400 \mathrm{kms}^{-1}$. Mahajan et al. (2011) worked with normalised velocities and found that backsplash fractions are approximately one third at projected radii slightly greater than the virial radius and $\left|v_{\text {los }}\right|<\sigma$. The fraction grows to one half if galaxies with $\left|v_{\text {los }}\right| \ll \sigma$ are considered. Based on these predictions, we select two samples of galaxies in the range $1<r / r_{\text {vir }}<2$ : galaxies having $\left|v_{\text {los }}\right|<0.5 \sigma$ (hereafter lowvelocity or LV) and galaxies with $\left|v_{\text {los }}\right|>\sigma$ (hereafter highvelocity or HV). Each of these samples has been divided into early type (ET) and late type (LT) using the criterion mentioned in Sect. 2.2. An important fraction of the galaxies in the LV sample are expected to have had one or more passes through the core of the cluster, while infalling galaxies should be common in the HV sample. The total number of galaxies considered in this work is 1060 . The number of galaxies on each subsample is quoted in Table 3.

\section{Results}

In Fig. 2 we compare the normalised distributions of the stellar mass $M_{*}$ for early-type galaxies. In the bottom panel of Fig. 2, we show the residuals between the pair of distributions: the difference $\Delta F(X)=f_{\mathrm{LV}}\left(M_{*}\right)-f_{\mathrm{HV}}\left(M_{*}\right)$, where $f_{\mathrm{LV}}\left(M_{*}\right)$ and $f_{\mathrm{HV}}\left(M_{*}\right)$ are the fraction of galaxies in the bin centred on $M_{*}$ in the LV and the HV sample, respectively. Based on a Kolmogorov-Smirnov test, we find that ET-LV galaxies are, on average, less massive than ET-HV at $>99 \%$ confidence level. This tendency is in agreement with Gill et al. (2005) who found that backsplash galaxies lose part of their mass in a single passage through the cluster centre. Nevertheless, it should be noted that Gill et al. (2005) used DM simulations and we are considering stellar masses.

Given that galaxy properties strongly depend on stellar mass (Kauffmann et al. 2003) and that LV and HV samples have different stellar mass distributions, we explore the dependence of age, metallicity, and the SFF as a function of the stellar mass for LV and HV galaxies. Figure 3 shows the median of age $\tau$ as a function of the stellar mass $\log \left(M_{*}\right)$. Error bars are obtained by the bootstrap resampling technique. The upper-left panel corresponds to the total sample while early and late are shown in the lower-left panel. For the total sample (LT+ET) we find that LV galaxies are older than HV; however, this tendency is nonstatistically significant. If morphological types are considered,

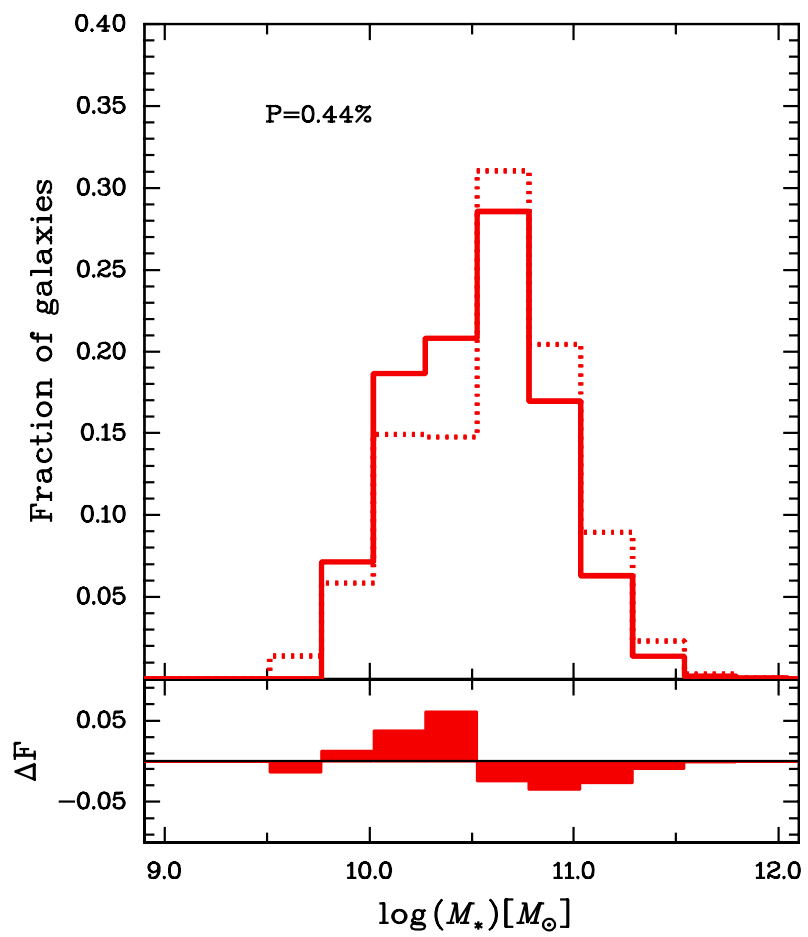

Fig. 2. Distributions of the stellar mass for early-type galaxies. The continuous line shows the LV galaxies, while HV galaxies are shown as a dotted line. The bottom panel shows the residuals between the distributions as shaded histogram.

we can see that age difference is only present for LT galaxies. Although for most of the bins the differences in age as a function of the stellar mass between LT-LV and LT-HV are nonstatistically significant, the reported trend is systematic and appears in nearly all bins. Except for the contamination produced by the error in the mass determination, each bin of stellar mass is independent; therefore, the systematic differences give confidence to the trend. In order to quantify the significance of this effect, we apply the following test. We compute the parameter $\Delta_{\mathrm{obs}}$,

$\Delta_{\text {obs }} \equiv \sum_{k=1}^{N_{\text {bin }}} \sum_{i=1}^{N_{\mathrm{A}}^{k}} \sum_{j=1}^{N_{\mathrm{B}}^{k}}\left(Y_{\mathrm{A}_{i}}^{k}-Y_{\mathrm{B}_{j}}^{k}\right) / \sum_{k=1}^{N_{\text {bin }}} N_{\mathrm{A}}^{k} N_{\mathrm{B}}^{k}$,

where $Y_{\mathrm{A}_{i}}^{k}$ is the value of the property $Y$ (e.g. the age $\tau$ ) and A denotes the sample (e.g. LV) of the $i$ th galaxy of a total $N_{\mathrm{A}}^{k}$ objects in the $k$ th bin of stellar mass; B denotes the second sample (e.g. $\mathrm{HV})$. Basically, $\Delta_{\text {obs }}$ represents the mean difference between two samples of galaxies once the trend of the galaxy property with the stellar mass is removed. The resulting values of $\Delta_{\text {obs }}$ for age are shown as vertical lines in the right-side panels of Fig. 3. In order to test how significant these values are, we implement the following test. For each bin of stellar mass, we create a sample of $N_{\mathrm{C}}^{k}$ galaxies composed of the sum of the $N_{\mathrm{A}}^{k}+N_{\mathrm{B}}^{k}$ galaxies in the $k$ th bin. From $N_{\mathrm{C}}^{k}$, we randomly select two samples of galaxies with $N_{\mathrm{A}}^{k}$ and $N_{\mathrm{B}}^{k}$ galaxies and compute $\Delta_{\text {boot }}$ as in Eq. (3). The procedure is repeated 1000 times. The resulting values of $\Delta_{\text {boot }}$ are shown in the right-side panels of Fig. 3. Finally, we compute the probability of having values of $\Delta_{\text {boot }}$ that depart from zero more than $\Delta_{\text {obs }}$. These $P$ values are quoted in the corresponding panels of Fig. 3. We can see that the differences in age between the LV and the HV samples are quite significant for the LT and the total sample (>99\%). Nevertheless, for the total 


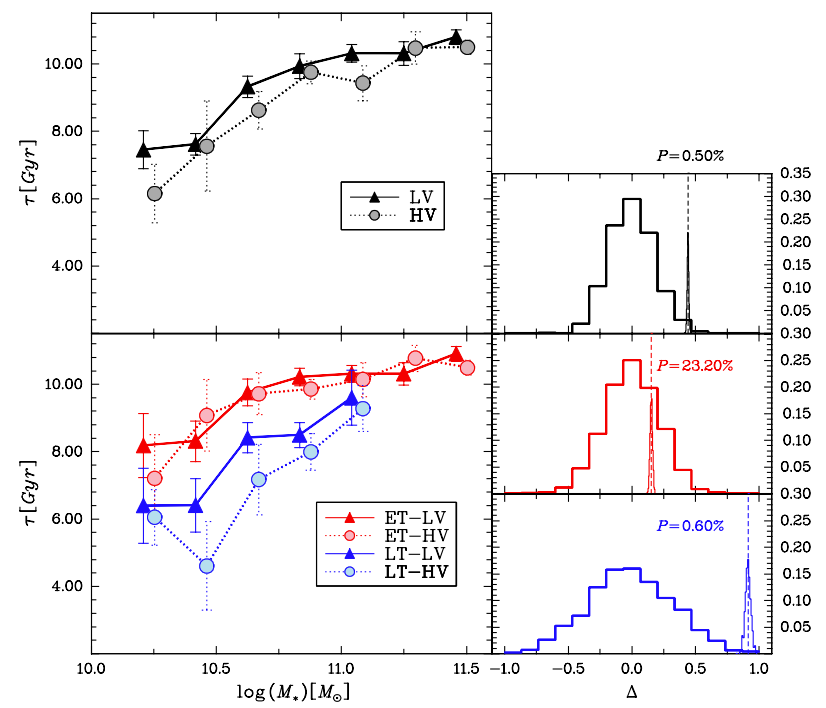

Fig. 3. Age as a function of stellar mass. Points represent the median in each bin, and vertical error bars are obtained by using the bootstrap resampling technique. Filled triangles and continuous lines represent the population of LV galaxies, while HV objects are shown as filled circles and dotted lines. The upper-left panel corresponds to the total sample of galaxies, while early-type (red) and late-type (blue) galaxies are shown in the bottom-left panel. HV galaxies have been shifted in 0.03 on the $x$-axis. Right-side panels show the confidence test (see text). Vertical dashed lines show $\Delta_{\text {obs }}$ value obtained by Eq. (3).

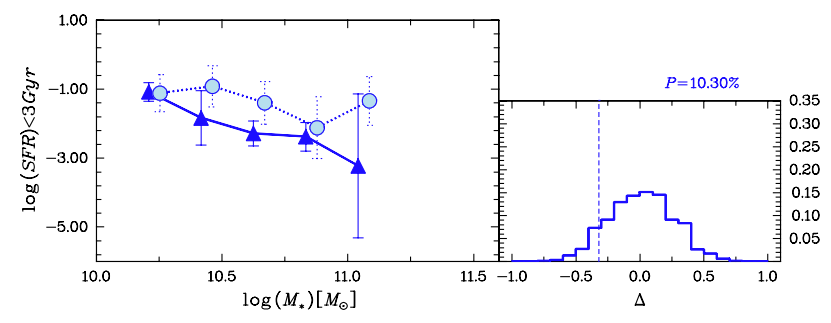

Fig. 4. Left-side panel: star formation fraction during the last 3 Gyrs as a function of stellar mass for LT galaxies. Points represent the median in each bin, and vertical error bars are obtained by using the bootstrap resampling technique. Right-side panel shows the confidence test (see text). Points and line types as in Fig. 3.

sample it should be noted that of the seven bins of stellar mass only three show differences in age, consequently the effect for the total sample should be considered very marginal. In order to understand how the uncertainties on the model-derived quantities affect our results, we implement the following test. Tojeiro \& Percival (2010) found that the average systematic error on an $8 \mathrm{Gyr}$ population is approximately $1 \mathrm{Gyr}$. For each galaxy in our sample we compute $\tau^{\prime}=\tau+\delta$, where $\delta$ is a random normally distributed error with a standard deviation of 1 Gyr. This new sample is used to compute $\Delta_{\text {obs }}^{\prime}$ using Eq. (3) and the procedure is repeated 1000 times. The results of this test are shown as a thin histogram in the right panels of Fig. 3. As can be observed, the uncertainty introduced by the systematic errors in the modelderived quantities are an order of magnitude smaller than the uncertainties produced by the cosmic variation (galaxy-to-galaxy).

No differences are found when the metallicity is considered. If the fraction of star formation during the last $3 \mathrm{Gyr}$ is considered, our analysis suggests (with a marginal significance $(\sim 90 \%))$ that the LT-HV galaxies have higher SFF than LTLV galaxies (see Fig. 4).

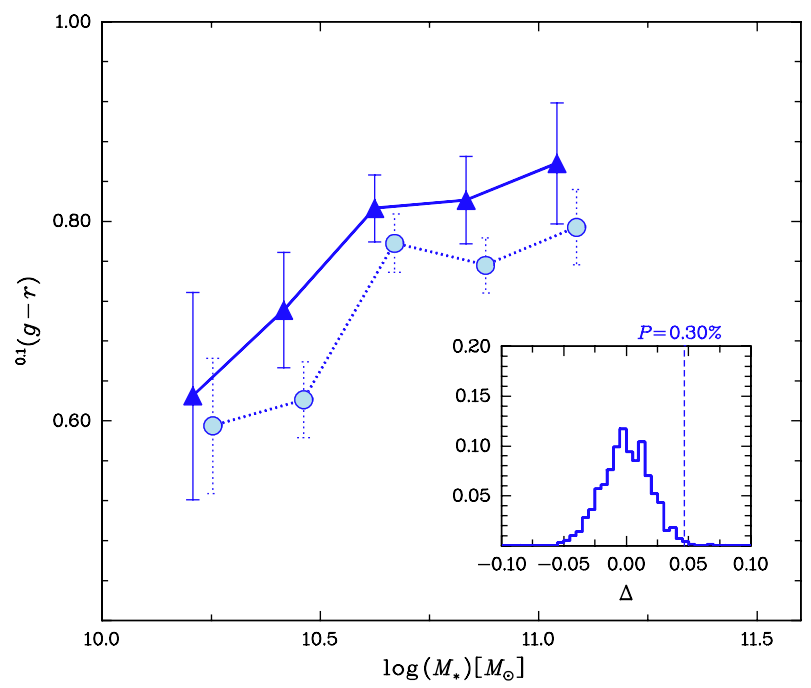

Fig. 5. ${ }^{0.1}(g-r)$ as a function of stellar mass for LT galaxies. Points represent the median in each bin. Vertical error bars are obtained by using the bootstrap resampling technique. The inset shows the confidence test (see text). Symbol types as in Fig. 3.

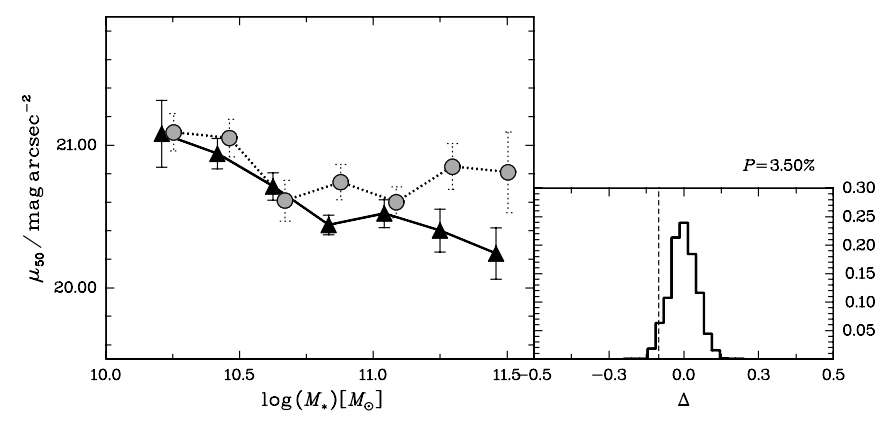

Fig. 6. Surface brightness $\mu_{50}$ as a function of the stellar mass for the total sample of galaxies. Points represent the median in each bin. Vertical error bars are obtained by using the bootstrap resampling technique. Right-side panel shows the confidence test (see text). Symbol types as in Fig. 3.

For the subsamples of LV and HV galaxies we also analyse the correlation between the stellar mass and several photometric properties. In Fig. 5 we plot the median of ${ }^{0.1}(g-r)$ as a function of $\log \left(M_{*}\right)$ for late-type galaxies. We find that LT-HV galaxies are bluer than LT-LV (>99\% confidence level). This effect is in agreement with the excess of recent star formation shown in Fig. 4. Figure 6 shows the median of the surface brightness $\mu_{50}$ as a function of $\log \left(M_{*}\right)$ for the total sample. We see signals at $96.5 \%$ confidence level in the sense that LV galaxies have higher surface brightness than HV galaxies. If the mean absolute magnitude of the LV and HV galaxies as a function of the stellar mass is compared, no difference is observed. Finally, in Fig. 7 we show the correlation between the Petrosian half-light radius $r_{50}$ as a function of $\log \left(M_{*}\right)$ for the total sample of galaxies. Although marginally significant, we can see that LV galaxies with $\log \left(M_{*} / M_{\odot}\right) \geqq 10.7$ are on average smaller than HV galaxies. The right panel of the figure shows the result of our test of significance, where we also include the same analysis for a subsamples of galaxies with $\log \left(M_{*} / M_{\odot}\right) \geqq 10.7$. The surface brightness and $r_{50}$ are not independent parameters; the lack of differences in absolute magnitude between LV and HV galaxies suggests that the differences in $\mu_{50}$ observed in Fig. 6 are due to the smaller mean size of $\mathrm{LV}$ galaxies with respect to HV galaxies. 
H. Muriel and V. Coenda: Galaxy properties in clusters. II.

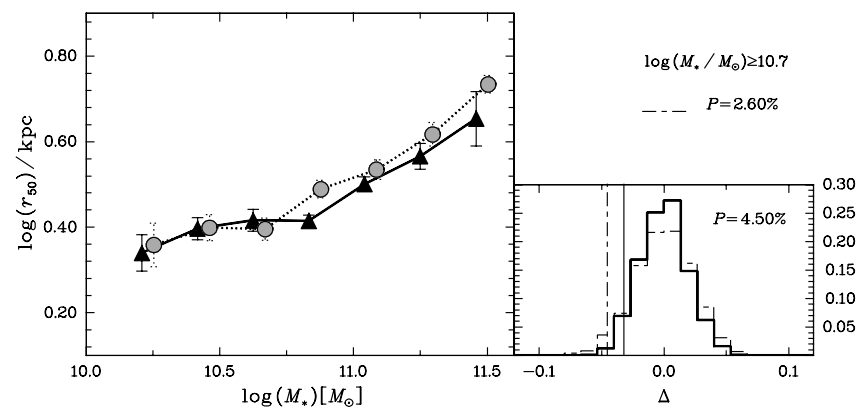

Fig. 7. Left-side panel: Petrosian half-light radius $r_{50}$ as a function of stellar mass for the total sample of galaxies. Points represent the median in each bin. Vertical error bars are obtained by using the bootstrap resampling technique. Right-side panel shows the confidence test for the total sample (continuous lines) and for a subsample of galaxies with $\log \left(M_{*}\right) \geqq 10.7$ (dash dotted lines). Symbol types as in Fig. 3 .

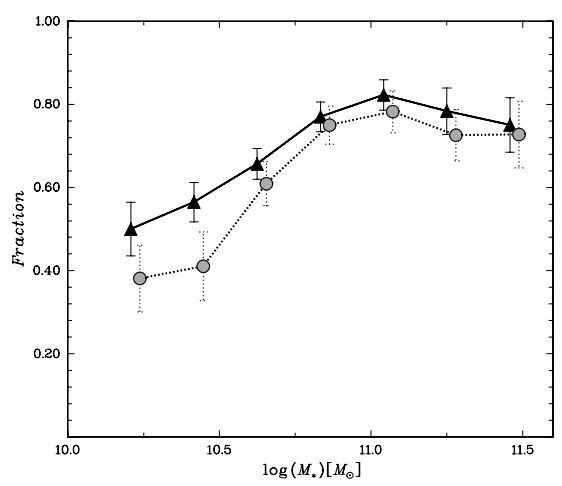

Fig. 8. Fraction of ETs as a function of the stellar mass. Points represent the median in each bin. Vertical error bars are obtained by using the bootstrap resampling technique. Symbol types as in Fig. 3.

For the whole sample of LV+HV galaxies, we find that the fraction of ETs grows with the stellar mass, going from $50 \%$ for the lowest mass bin up to $80 \%$ for high-mass galaxies. We test whether these fractions depend on the subsample of galaxies. We find that, for all the mass bins considered in this work, the fraction of ETs is larger in the LV sample than in the HV (see Fig. 8). The difference is larger for low-mass galaxies, where the fraction of ETs in the LV sample is up to $35 \%$ larger than in the HV sample. For the high-mass bins, this difference falls to $5 \%$. Using the same test previously discussed, we find that the LV and HV samples have different fraction of ETs at the $98 \%$ confidence level.

\section{Conclusions and discussion}

Based on a sample of galaxy clusters composed of 90 X-ray selected and MaxBCG clusters in the redshift range $0.05<$ $z<0.10$, we analyse properties of 1060 SDSS galaxies on the outskirts of clusters $\left(1<r / r_{\text {vir }}<2\right)$. Using the radial velocity of galaxies (relative to the mean cluster velocity), we select low- and high-velocity subsamples as follows: $\left|v_{\text {los }}\right|<0.5 \sigma$ and $\left|v_{\text {los }}\right|>\sigma$, respectively. According to theoretical predictions, a significant fraction of the first subsample should be backsplash galaxies, that is, objects that have already orbited near the cluster centre. In the backsplash scenario, the sample of galaxies with high relative velocity would include a significant fraction of objects that are falling into the cluster for the first time. We analyse two sets of properties, i) photometric properties colour ${ }^{0.1}(g-r)$, the Petrosian $r$-band absolute magnitude $M_{0.1} r$, the radius that encloses $50 \%$ of the Petrosian flux, and the $r$-band surface brightness computed inside $r_{50}-$ and ii) properties derived from the spectral synthesis analysis; we use the VESPA algorithm (Tojeiro et al. 2009) to compute the stellar mass $M_{*}$, the mean age $\tau$ weighted by mass, the metallicity $Z$, and the fraction of the stars that were formed during the last 3 Gyrs.

We find that ET-LV galaxies are systematically less massive than ET-HV, in agreement with the predictions of Gill et al. (2005) who found clear evidence that LV galaxies lose part of their mass in a single passage through the cluster centre. For the whole sample of galaxies, we find that the fraction of ETs grows with the stellar mass, going from $50 \%$ for the lowest mass bin up to $80 \%$ for high mass galaxies. The fraction of ETs was larger in the LV sample than in the HV. The difference was larger for lowmass galaxies, in which the fraction of ETs in the LV sample was up to $35 \%$ larger than in the HV sample. This result supports the scenario in which the central region of clusters of galaxies can contribute to the transformation of morphological types.

Galaxies with the same stellar mass and in the same environment are expected to be similar. Departures from the mean properties may be directly linked to differences in the recent dynamical history, as would be the case when comparing LV and HV galaxies with the same stellar mass in the same cluster region. We test this hypothesis for each of the galaxy properties considered in our work. Our results show that, for the same stellar mass, LV galaxies are on average older than HV. If morphological types are considered, we find that this effect corresponds to LT galaxies. These results are consistent with the age gradients found in clusters by Rakos et al. (2007). Smith et al. (2012) found that the age gradient depends on the mass of galaxy being lower for the most massive objects. These authors suggested that, even if there is a significant pre-processing in groups, the location in the cluster core at $z=0$ effectively selects galaxies which passed through all stages of the environmental history, including pre-processing, earlier than those which today reside in the cluster outskirts (see also Price et al. 2011). Within this scenario, LV are more advanced in the process of accretion and should therefore be, on average older, as observed in our analysis.

We find some evidence that LT-HV galaxies have formed more stars during the last 3 Gyrs than LT-LV galaxies, consistent with Mahajan et al. (2011) and with models that assume that a passage through the central region of clusters tends to quench star formation because of processes like ram pressure stripping or strangulation. This phenomenon is also reflected in the colours of galaxies. Our results show that LT-LV galaxies are less blue (have had less recent star formation) than LT-HV.

Although marginally significant, we find that LV galaxies with $\log \left(M_{*} / M_{\odot}\right) \geqq 10.7$ tend to have a higher surface brightness than HV. Analysing the size and the luminosity of galaxies, our results suggest that the higher values of $\mu_{50}$ for $L V$ galaxies is likely to be due to the smaller size of LV galaxies (for a given stellar mass) in comparison to HV galaxies. These results could be related to gravitational-type processes, in which galaxies that have orbited the central regions of clusters are more likely to suffer tidal effects, such as the loss of part of their mass as well as a redistribution of matter towards more compact configurations. This result is in agreement with Aguilar \& White (1986) who used $N$-body simulations to find that strong collisions result in a reduced effective radius and a higher surface density.

Acknowledgements. This work has been partially supported with grants from Consejo Nacional de Investigaciones Científicas y Técnicas de la República Argentina (CONICET) and Secretaría de Ciencia y Tecnología de la Universidad de Córdoba. We thank the anonymous referee for a careful reading and useful 
questions and suggestions that improved the presentation of this paper. We would like to thank Marcelo Lares for helpful discussions about the error analysis. Funding for the Sloan Digital Sky Survey (SDSS) has been provided by the Alfred P. Sloan Foundation, the Participating Institutions, the National Aeronautics and Space Administration, the National Science Foundation, the US Department of Energy, the Japanese Monbukagakusho, and the Max Planck Society. The SDSS web site is http://www.sdss.org/. The SDSS is managed by the Astrophysical Research Consortium (ARC) for the Participating Institutions. The Participating Institutions are The University of Chicago, Fermilab, the Institute for Advanced Study, the Japan Participation Group, The Johns Hopkins University, the Korean Scientist Group, Los Alamos National Laboratory, the Max Planck Institut für Astronomie (MPIA), the Max Planck Institut für Astrophysik (MPA), New Mexico State University, University of Pittsburgh, University of Portsmouth, Princeton University, the United States Naval Observatory, and the University of Washington.

\section{References}

Abadi, M. G., Moore, B., \& Bower, R. G. 1999, MNRAS, 308, 947

Abazajian, K. N., Adelman-McCarthy, J. K., Agüeros, M. A., et al. 2009, ApJS, 182,543

Adelman-McCarthy, J. K., Agüeros, M. A., Allam, S. S., et al. 2007, ApJS, 172, 634

Aguerri, J. A. L., \& Sánchez-Janssen, R. 2010, A\&A, 521, A28

Aguilar, L. A., \& White, S. D. M. 1986, ApJ, 307, 97

Beers, T. C., Flynn, K., \& Gebhardt, K. 1990, AJ, 100, 32

Blanton, M. R., Brinkmann, J., Csabai, I., et al. 2003, AJ, 125, 2348

Blanton, M. R., Eisenstein, D., Hogg, D. W., Schlegel, D. J., \& Brinkmann, J. 2005, ApJ, 629, 143

Bohringer, H., Voges, W., Huchra, J. P., et al. 2000, VizieR Online Data Catalog, 212, 90435

Bruzual, G., \& Charlot, S. 2003, MNRAS, 344, 1000

Coenda, V., \& Muriel, H. 2009, A\&A, 504, 347

De Lucia, G., Weinmann, S., Poggianti, B. M., Aragón-Salamanca, A., \& Zaritsky, D. 2012, MNRAS, 423, 1277

Deng, X.-F. 2013, Res. Astron. Astrophys., 13, 651

Díaz, E., Zandivarez, A., Merchán, M. E., \& Muriel, H. 2005, ApJ, 629, 158

Dressler, A. 1980, ApJS, 42, 565

Ellison, S. L., Simard, L., Cowan, N. B., et al. 2009, MNRAS, 396, 1257
Fujita, Y., \& Nagashima, M. 1999, ApJ, 516, 619

Gill, S. P. D., Knebe, A., \& Gibson, B. K. 2005, MNRAS, 356, 1327

Gunn, J. E., \& Gott, J. R. I. 1972, ApJ, 176, 1

Huchra, J. P., \& Geller, M. J. 1982, ApJ, 257, 423

Hyde, J. B., \& Bernardi, M. 2009, MNRAS, 349

Kauffmann, G., Heckman, T. M., White, S. D. M., et al. 2003, MNRAS, 341, 54

Koester, B. P., McKay, T. A., Annis, J., et al. 2007, ApJ, 660, 239

Larson, R. B., Tinsley, B. M., \& Caldwell, C. N. 1980, ApJ, 237, 692

Mahajan, S. 2013, MNRAS, 431, L117

Mahajan, S., Mamon, G. A., \& Raychaudhury, S. 2011, MNRAS, 416, 2882

Maraston, C. 2005, MNRAS, 362, 799

McCarthy, I. G., Frenk, C. S., Font, A. S., et al. 2008, MNRAS, 383, 593

Moore, B., Lake, G., \& Katz, N. 1998, ApJ, 495, 139

Moore, B., Lake, G., Quinn, T., \& Stadel, J. 1999, MNRAS, 304, 465

Neistein, E., van den Bosch, F. C., \& Dekel, A. 2006, MNRAS, 372, 933

Park, C., \& Choi, Y.-Y. 2005, ApJ, 635, L29

Petrosian, V. 1976, ApJ, 209, L1

Pimbblet, K. A., Smail, I., Edge, A. C., et al. 2006, MNRAS, 366, 645

Poggianti, B. M., Fasano, G., Bettoni, D., et al. 2009, ApJ, 697, L137

Popesso, P., Böhringer, H., Brinkmann, J., Voges, W., \& York, D. G. 2004, A\&A, 423, 449

Price, J., Phillipps, S., Huxor, A., Smith, R. J., \& Lucey, J. R. 2011, MNRAS, 411, 2558

Rakos, K., Schombert, J., \& Odell, A. 2007, ApJ, 658, 929

Rasmussen, J., Mulchaey, J. S., Bai, L., et al. 2012, ApJ, 757, 122

Rines, K., Geller, M. J., Kurtz, M. J., \& Diaferio, A. 2005, AJ, 130, 1482

Schlegel, D. J., Finkbeiner, D. P., \& Davis, M. 1998, ApJ, 500, 525

Schmidt, M. 1968, ApJ, 151, 393

Shimasaku, K., Fukugita, M., Doi, M., et al. 2001, AJ, 122, 1238

Smith, R. J., Lucey, J. R., Price, J., Hudson, M. J., \& Phillipps, S. 2012, MNRAS, 419,3167

Strateva, I., Ivezić, Ž., Knapp, G. R., et al. 2001, AJ, 122, 1861

Strauss, M. A., Weinberg, D. H., Lupton, R. H., et al. 2002, AJ, 124, 1810

Tojeiro, R., \& Percival, W. J. 2010, MNRAS, 405, 2534

Tojeiro, R., Heavens, A. F., Jimenez, R., \& Panter, B. 2007, MNRAS, 381, 1252

Tojeiro, R., Wilkins, S., Heavens, A. F., Panter, B., \& Jimenez, R. 2009, ApJS, 185,1

Wilman, D. J., Oemler, Jr., A., Mulchaey, J. S., et al. 2009, ApJ, 692, 298

York, D. G., Anderson, Jr., J. E., Anderson, S. F., et al. 2000, AJ, 120, 1579 